\title{
Transition from Bosentan to Ambrisentan in Pulmonary Arterial Hypertension: A Single-Center Prospective Study
}

\author{
Jingyuan Chen \\ Jun Luo \\ Xiaojie Yang \\ Peng Luo \\ Yusi Chen \\ Zilu Li (D) \\ Jiang Li (D)
}

Department of Cardiovascular Medicine, Second Xiangya Hospital, Central South University, Changsha City, Hunan

Province, People's Republic of China
Correspondence: Jiang Li

Department of Cardiovascular Medicine, Second Xiangya Hospital, Central South University, Changsha City, Hunan

Province, People's Republic of China

Email lijiangcs@csu.edu.cn
Background: Pulmonary hypertension patients experienced a high financial burden due to the high cost of drug therapy, high incidence of comorbidities and hospitalizations. Endothelin receptor antagonists (ERAs) in PAH treatment showed a high cost. While ambrisentan has been covered by medical insurance of a local government of China, there has been a drug transition from bosentan to ambrisentan in treating PAH patients. We evaluated the safety, efficacy and tolerability of ambrisentan after drug transition.

Methods: Liver and renal functions were inspected at baseline, month 1, 3 and 6. NTproBNP, echocardiographic variables, WHO functional class (WHO-FC), 6-minute walking distance (6MWD) were measured in the baseline and month 6 to evaluate the safety and efficacy. Quality of life (QOL) scale was used in the baseline and month 6 to investigate the tolerability and quality of life of PAH patients.

Results: Among 224 PAH patients, 49 stable PAH patients meet the inclusion criteria were enrolled, among which three patients discontinued during the study. Our results showed no difference in 6-minute walking distance (6MWD) of PAH patients from baseline and month 6. The liver and renal function, N-terminal pro-brain natriuretic peptide (NT-proBNP), WHO functional class (WHO-FC) showed no difference either. For echocardiography parameters, the left ventricular end-diastolic dimension (LVEDD) of month 6 decreased. Other parameters were no significant difference from the baseline. There was no difference in the QOL scale between baseline and month 6 .

Conclusion: Our results suggested that it is safe and tolerable for stable PAH patients to transition from bosentan to ambrisentan without influencing hematologic parameters or heart function.

Keywords: pulmonary arterial hypertension, ambrisentan, bosentan, endothelin receptor antagonist, drug transition

\section{Introduction}

Pulmonary arterial hypertension (PAH) is a chronic and devastating disease characterized by progressively increasing pulmonary artery pressure and vascular resistance that results in right heart failure and death. Despite multiple treatment options, the prognosis of pulmonary arterial hypertension (PAH) remains poor. ${ }^{1-3}$

Transition to pulmonary arterial hypertension (PAH)-specific drugs is considered in patients with PAH who do not respond to a therapeutic pathway or who experience side effects to the combination of drugs. Several studies have compared the transition of medicines from one pathway to another. However, little is known 
about the transition between the same category, especially in the endothelin receptor antagonists (ERAs). ${ }^{4}$

Due to the high cost of drug therapy, high incidence of comorbidities, hospitalizations, PAH patients experience a high financial burden. ${ }^{5}$ In western countries, most medication therapies were covered by medical insurance. However, for the full treatment of PAH, like initial combination therapy and additional optimized therapy for patients, whether or not covered by medical insurance, are still under debate.

In China, although treatment decisions followed by the guidelines, they are also influenced largely by the patients' financial situation. ${ }^{6,7}$ Although guidelines recommended initial combination therapies and there were lots of effective drugs such as macitentan and selexipag, most Chinese PAH patients tend to continue their monotherapy of ERAs or phosphodiesterase type 5 inhibitors (PDE5-i) because of financial reason.

Compared with other cardiovascular diseases, PAH patients have a lower onset age, heavier financial burden and higher mortality. With the development of medical and health care organizations in China, people have paid more attention to the disease and some local governments are now including it in medical insurance coverage.

Bosentan and ambrisentan are ERAs both expensive in China. On August 1, 2018, only ambrisentan has been added to the list of medical insurance in Hunan province. After being reimbursed for about $60-70 \%$ by medical insurance, monthly out-of-pocket expenses of ambrisentan is about US\$123-152, while that of bosentan is US\$576. As a result, there is a need for patients who were taking bosentan to transit to ambrisentan. There have been few studies on the transition between ERAs, and most of them were from bosentan or ambrisentan to macitentan. ${ }^{8-10}$ However, the effect of transition from bosentan to ambrisentan needs to be investigated, and the present study aimed to address this question.

\section{Methods}

\section{Trial Design}

This prospective, single-center, open-label study was conducted at Second Xiangya Hospital of Central South University, Changsha, Hunan Province, China, to assess the effects of the transition from bosentan to ambrisentan therapy on efficacy, safety and tolerability, and quality of life, for 6 months after the transition in patients with PAH.
All patients in this study received ambrisentan orally, $5 \mathrm{mg}$ once daily in the first 4 weeks. After measurements of liver and renal function, drug dose was adjusted to $10 \mathrm{mg}$, once daily after 4 weeks basing on the physicians' advice and patients' choice. Concomitant treatment with PDE5i and/or prostanoids was permitted. Dose reduction was permitted at any time for ambrisentan intolerance. Discontinuation was allowed at any point based on the physicians' judgment.

The primary endpoint for efficacy is 6-min walk test. Secondary endpoints include WHO-FC, echocardiography measurements, aminotransferase level, N-terminal probrain natriuretic peptide (NT-proBNP), echocardiography, electrocardiograph (ECG), and quality of life (QOL) scale.

The study's protocol and ethics were approved by the ethics committee of the Second Xiangya Hospital of Central South University and was conducted in accordance with the Declaration of Helsinki. All patients who participated in this program agreed with the protocol and signed informed consent before study entry. The trial was registered at Chinese Clinical Trial Registry, www.chictr.org.cn (No. ChiCTR1900025197).

\section{Patients}

Patients older than 18 years with idiopathic PAH (IPAH), PAH associated with congenital heart disease (CHD-PAH) or connective tissue disease (CTD-PAH), and chronic thromboembolic pulmonary hypertension (CTEPH) were enrolled. Eligibility criteria were defined to enroll stable patients whose disease was reasonably well controlled and at a mild to moderate risk stratification; World Health Organization (WHO) cardiac function class was from II to III. All patients must have been receiving bosentan and on a stable dose for at least 6 months before study entry. Concomitant PAH-specific therapies, including prostanoids, sildenafil, tadalafil and riociguat, were allowed if patients were already receiving these therapies for more than 6 months and were on a stable dose. There were no patients with liver or renal dysfunction above 3 times the upper limit of normal. The exclusion criteria included the following: acute heart failure, need for emergency care, high-risk stratification of disease, left heart diseaseassociated pulmonary hypertension, acute or chronic infection, any factor that might interfere with treatment compliance, or any known concomitant life-threatening disease with a life expectancy of below 6 months. Patients were screened for eligibility up to 14 days before study entry. 


\section{Patient Assessments}

Patients were followed up at month 1, 3 and 6. For safety measurement, intolerance symptoms and side effects could be reported at any time. All patients had blood drawn for liver and renal function tests and myocardial enzymes at month 1, 3 and 6. If liver or function tests were greater than 3 times the upper limit of normal or abnormal myocardial enzyme results, patients would quit the study. Efficacy assessments were conducted at baseline and month 6, including 6-minute walk distance (6MWD), WHO function class, NT-proBNP levels, and echocardiography. Echocardiographic parameters included the diameter of the atrium, ventricle, pulmonary artery and tricuspid annular plane systolic excursion (TAPSE). QOL and clinical status were assessed at baseline and month 6 to obtain the patient's perception of treatment effectiveness and convenience and global satisfaction. ${ }^{11}$

\section{Statistical Analysis}

Continuous variables (6MWD and echocardiography parameters) were summarized using mean and standard deviation (normal distribution) or quartile (non-normal distribution). Normally distributed data were compared using the paired Student $t$-test and non-normal distribution variables were compared by the Mann-Whitney $U$-test. Categorical variables (WHO functional class, response to QOL, and disease classification) were summarized using the number and percentage of patients in each category were compared using the chi-square test.

\section{Results}

\section{Patient Characteristics}

Among $224 \mathrm{PAH}$ who wished to transition, 15 were less than 18 years old, 54 were not in a mild to moderate risk stratification, 21 had abnormal renal or liver dysfunction. In the remaining 134 patients, only 49 patients were received and stable on their target therapy plan for more than 6 months (Figure 1). These 49 patients were prospectively enrolled from October 2018 to August 2019. Patients who met the study entry criteria were switched from bosentan to ambrisentan. The demographic and baseline disease characteristics of the patients are summarized in Table 1 . The majority of patients (58.7\%) were CHDPAH, followed by IPAH and CTD-PAH, at $28.3 \%$ and $10.9 \%$, respectively. All of these patients were in a stable state with WHO function classes II and III and a mild to moderate risk stratification. Most of these patients were

\section{PAH patients}

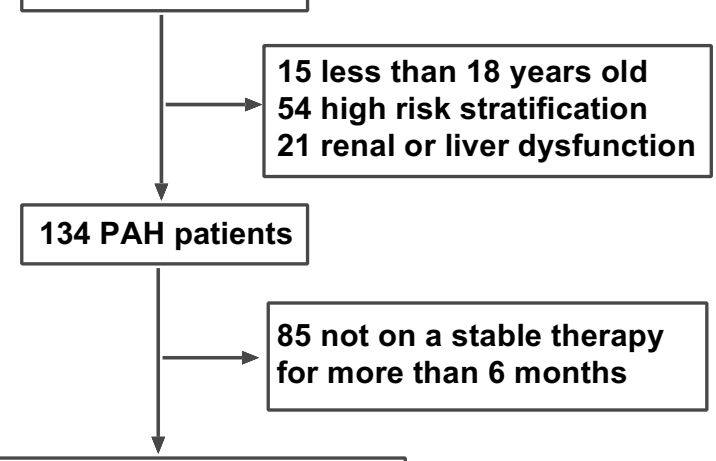

49 patients started the transition

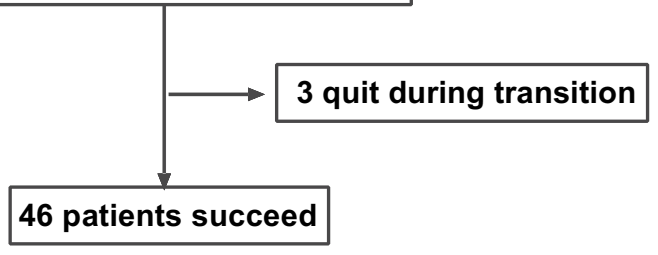

Figure I Flow chart of patient selection.

receiving combination therapy, in which $63 \%$ were receiving bosentan and sildenafil, $21.7 \%$ were receiving bosentan and tadalafil, $4.3 \%$ were receiving bosentan and

Table I Patient Demographics and Characteristics

\begin{tabular}{|l|l|}
\hline Baseline Characteristics & \\
\hline Age (y) & $34.4 \pm 9.5$ \\
\hline $\begin{array}{l}\text { Gender, } n \text { (\%) } \\
\text { Memale }\end{array}$ & $33(71.7)$ \\
\hline Etiology, $n$ (\%) & $13(28.3)$ \\
IPAH & \\
CHD-PAH & $13(28.3)$ \\
CTD-PAH & $27(58.7)$ \\
CTEPH & $5(10.9)$ \\
\hline WHO function class, $n$ (\%) & $1(2.2)$ \\
II & \\
III & $26(56.5)$ \\
\hline BMI (kg/m ${ }^{2}$ ) & $20(43.5)$ \\
\hline Drug therapy, n (\%) & $20.31 \pm 2.84$ \\
Bosentan monotherapy & \\
Bosentan and sildenafil & $5(10.9)$ \\
Bosentan and tadalafil & $29(63.0)$ \\
Bosentan and riociguat & $10(21.7)$ \\
\hline Time to take bosentan before transition (months) & $2(4.3)$ \\
\hline
\end{tabular}

Abbreviations: IPAH, idiopathic pulmonary artery hypertension; CHD-PAH, congenital heart disease-related pulmonary artery hypertension; CTD-PAH, connective tissue disease-related pulmonary artery hypertension; CTEPH, chronic thromboembolic pulmonary hypertension; BMI, body mass index. 
riociguat. Baseline $6 \mathrm{MWD}$ was $416 \pm 50 \mathrm{~m}$. Before transition, all patients were taking bosentan for more than 6 months. The median duration for taking bosentan of these patients was 12 months (from 6 to 83 months).

\section{Failure and Success of Drug Transition}

In the first 4 weeks of transition from bosentan, no patient had abnormal liver function (indicated by parameters more than 3 times of the upper limit of normal). There was also no significant dysfunction of myocardial enzymes and renal function.

In the following 5 months, three patients failed drug transition. One of them was because of side effects; another was because of the representation of symptoms. The last one was due to clinical deterioration and requiring in-hospital initiation of intravenous treprostinil.

For side effect, irregular and prolonged menstruation occurred in one female patient despite improvements in 6MWD, NT-proBNP and echocardiographic parameters of RV function. Menstruation irregularities improved after transitioning back to bosentan. Another patient had chest pain, dyspnea and sleeplessness after switching to ambrisentan for 2 months and felt these symptoms were influencing his daily life. Although not changed in 6MWD, NT-proBNP, ECG and not showing a worsening in echocardiography parameters, this patient decided to quit transition.

\section{Safety and Efficacy}

Forty-six out of 49 patients went through the drug transition. Large amounts of patients had side effects after taking ERAs. Before conversion to ambrisentan, $60.9 \%$ of patients taking bosentan had side effects. And, $67.9 \%$ of them had one to two side effects. The most common adverse effects of bosentan were dizziness, headache and flushing, accounting for $42.9 \%, 35.7 \%$ and $35.7 \%$, respectively. After the transition, $58.7 \%$ of patients had side effects. Dizziness (44.4\%), headache (37.0\%), and palpitation $(33.3 \%)$ were the most common. Despite a high incidence, most side effects were mild to moderate. Most patients assumed those side effects were well tolerated and would not influence their work or daily lives. We did not observe any substantial differences in the side effects profile before or after transition.

There were no significant differences in the function class, exercise capacity or echocardiography characteristics before and after transition (Table 2). For the assessment of exercise capacity, from baseline and month 6, the 6MWD remained unchanged, from $416 \pm 50 \mathrm{~m}$ to 427
Table 2 Baseline and Follow-Up Data of Exercise Capacity, Heart Function and Quality of Life

\begin{tabular}{|c|l|l|l|}
\hline & Baseline & Month 6 & P \\
\hline $\begin{array}{c}\text { Echocardiography } \\
\text { RA (mm) }\end{array}$ & $40.00(36.00-46.00)$ & $39.00(35.00-46.00)$ & 0.592 \\
RV (mm) & $42.39 \pm 9.78$ & $41.26 \pm 9.84$ & 0.582 \\
LA (mm) & $30.50(28.00-33.25)$ & $32.00(29.00-35.00)$ & 0.207 \\
LVEDD (mm)* & $37.65 \pm 4.58$ & $40.26 \pm 5.07$ & 0.011 \\
PA (mm) & $30.00(27.00-35.00)$ & $29.00(27.00-33.00)$ & 0.641 \\
TAPSE (mm) & $15 \pm 3$ & $16 \pm 3$ & 0.175 \\
\hline 6MWD (m) & $416 \pm 50$ & $427 \pm 48$ & 0.270 \\
\hline NT-proBNP (pg/mL) & $427.50(131.57-953.46)$ & $301.13(112.21-683.63)$ & 0.223 \\
\hline $\begin{array}{l}\text { WHO function } \\
\text { class, } n(\%)\end{array}$ & & & 0.454 \\
I & 0 & $1(2.17)$ & \\
II & $26(56.5)$ & $28(60.87)$ & \\
\hline III & $20(43.5)$ & $17(36.96)$ & \\
\hline \begin{tabular}{l} 
Quality of life scale \\
\hline
\end{tabular} & $83 \pm 6$ & $84 \pm 7$ & 0.208 \\
\hline
\end{tabular}

Note: $* P<0.05$.

Abbreviations: RA, right atrium; RV, right ventricle; LA, left atrium; LVEDD, left ventricular end-diastolic dimension; PA, pulmonary artery; TAPSE, tricuspid annular plane systolic excursion; 6MWD, 6 minutes walking distance; NT-proBNP, $\mathrm{N}$-terminal pro-brain natriuretic peptide.

$\pm 48 \mathrm{~m}$ (Figure 2). For echocardiography parameters, the area of right atrium (RA), right ventricle (RV), left atrium (LA) and pulmonary artery (PA) width, as well as TAPSE, which for the evaluation of RV function, showed no significant differences. Left ventricular end-diastolic dimension (LVEDD) was increased from $37.65 \pm 4.58$ to $40.26 \pm 5.07$ $(\mathrm{P}=0.011)$.

In parameters of heart function, NT-proBNP showed no difference between baseline and month 6 after the

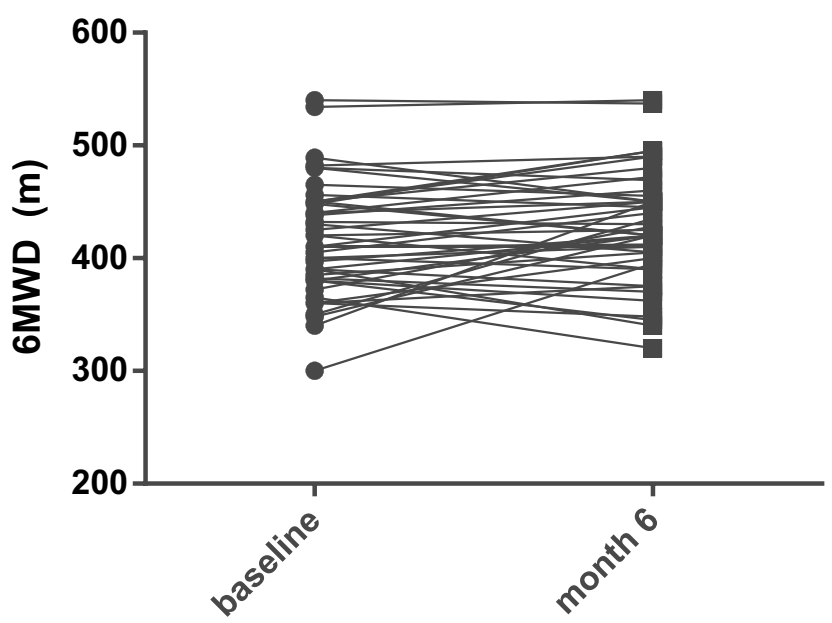

Figure 2 6MWD change from baseline to month $6(n=46)$. Left of each line was 6MWD in baseline and the right of each line was 6MWD of the same person in month 6. 6MWD: 6-minute walking distance. 
transition, from 427.50 (131.57-953.46) to 301.13 (112.21-683.63) $(\mathrm{P}=0.223)$. Besides, for the WHO cardiac function class, there was no significance observed between baseline (no class I, $56.50 \%$ of class II, and $43.50 \%$ of class III) and month 6 (class I $2.17 \%$, class II $60.87 \%$ and class III 36.96\%) $(\mathrm{P}=0.454)$ (Table 2 and Figure 3$)$.

\section{Satisfaction and Quality of Life}

Treatment satisfaction data were collected for 46 patients by using the QOL scale and were not available for 3 patients as they quit in the month 6. QOL questionnaire is composed of 6 contents, which are treatment effectiveness, convenience of use, impact on daily living/activities, global satisfaction and side effects. ${ }^{11}$ The mean \pm SD for the QOL scale was $83 \pm 6$ at baseline and $84 \pm 7$ at month 6 after the transition of ambrisentan and were no significant differences. There were also no differences between the items of treatment effectiveness, impact on daily living/ activities global satisfaction and side effects. However, most of the patients thought it was more convenient for taking once daily of ambrisentan $(\mathrm{P}<0.001)$.

\section{Discussion}

Several clinical trials have been conducted to optimize drug therapies in PAH. Along with the progress in investigating PAH targeted therapies, the guidelines recommended initial combination therapy and sequential combination therapy. However, drug combination and transition have generated some issues. ${ }^{12,13}$ There are several underlying reasons for drug transition, such as side effects, using convenience, using level of evidence, financial burden and so on. ${ }^{4}$ In the drug transition researches to date, the majority studied the transition in prostaglandins, for the reason of different methods of administration and frequency. ${ }^{14-18}$ For ERAs, it showed sitaxsentan may represent a safe and efficacious alternative ERA for patients discontinuing bosentan because of liver function abnormalities. ${ }^{19}$ Meanwhile, the evidence found in

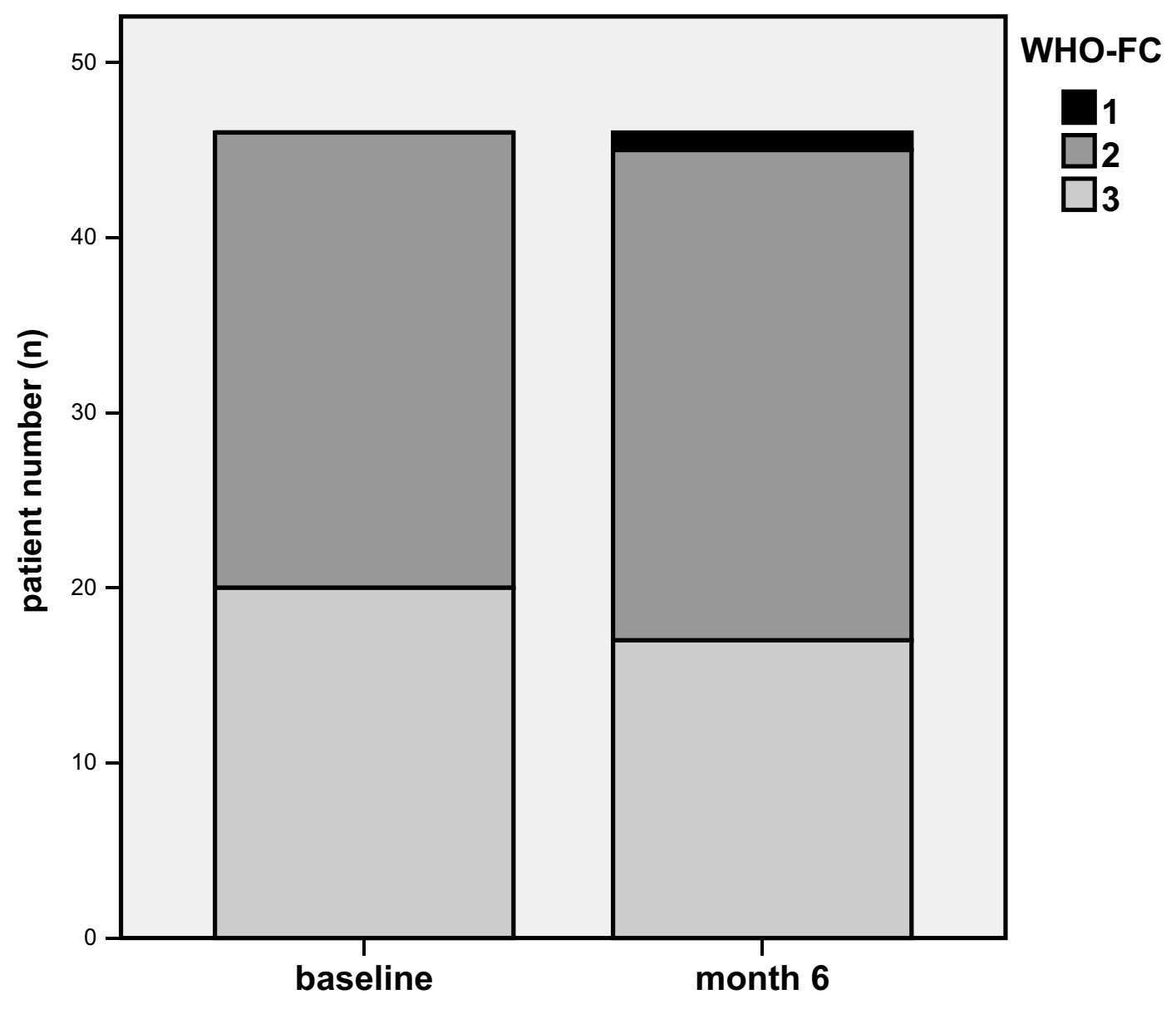

Timepoint

Figure 3 The number of patients in different WHO function class (WHO-FC) in baseline and month 6. 
patients with liver abnormalities due to bosentan or sitaxentan, the transition to ambrisentan appears to be safe and can result in clinical improvement. ${ }^{20}$ The other two studies, as measured by NT-proBNP, echocardiography, hemodynamic parameters, as well as side effects, also found that switch from sitaxsentan to bosentan or ambrisentan was safe and tolerable. ${ }^{21,22}$ Another prospective study has observed only eight patients who transitioned to ambrisentan after taking bosentan for 1 year and there are no differences between the transition patients and those who are still taking ambrisentan. ${ }^{23}$ This study was the first to investigate the safety, efficacy and satisfaction of PAH patients in transition from bosentan to ambrisentan and was in patients without liver abnormalities. The main reason for drug transition is the financial burden of patients. Ambrisentan was covered by medical insurance of local government, and out-of-pocket costs of ambrisentan are only a quarter of bosentan.

After the transition from bosentan $125 \mathrm{mg}$ twice daily to ambrisentan $5 \mathrm{mg}$ once daily in the first 4 weeks, no patients had abnormalities in the liver, renal functions or cardiac enzymes. After another 5 months, patients taken ambrisentan showed a well adaptation of drug transition. There were no differences in side effects between the baseline and 6 months after drug transition. Overall, there were no significant differences in 6MWD, WHO function class and echocardiography parameters except for LVEDD in patients who completed the transition. As these patients were administered bosentan for more than 6 months, and all patients have maintained the risk stratification of a mild to moderate class. There was a decreased tendency of NT-proBNP, and improvement for LVEDD, which in accordance with the prior study which reported the transition from sitasentan to ambrisentan. ${ }^{20}$

For the majority of patients, 6MWD and WHO function class and risk stratification remained the same. Although not much, there were still some patients who deteriorated from FC II to FC III. We considered it might relate to the disease progression and it was in accordance with other drug transition experiments. ${ }^{17,20,21}$

As with the results of efficacy and safety, there were no differences in satisfaction before and after the drug transition. Although in the convenience category, patients identified the convenience of ambrisentan for reduced pill burden and frequency of dosing, there was no significant difference in the general satisfaction. The reasons for this result might relate to the sample size and the worries of the drug transition itself.
As for side effects, although most patients experienced some side effects, there were no differences in the occurrence rate of the side effects before and after the transition. When taking bosentan, most of the side effects were dizziness, headache and flushing. After the transition, the majority of the side effects were dizziness, headache and palpitation. All patients considered these side effects were mild to moderate and would not influence their daily life.

Limitations of this study included the open-label nature of the study and the small sample size. In our study, not all patients were adjusted to $10 \mathrm{mg}$ daily of ambrisentan after 4 weeks, which might influence results of efficacy. Awareness of the transition might create bias by generating a perceived improvement from the transition. Compared with other transition studies, the lack of RHC parameters in this study was not powerful enough for efficacy assessment. Our results showed that the transition from bosentan to ambrisentan was safe and tolerable. It also demonstrated feasibilities for those who have the need to change their specific treatment strategies.

In conclusion, there were no significant differences in renal or liver function of ambrisentan treatment and might have consistency with the exercise capacity. Our results suggested that it is safe and tolerable for stable $\mathrm{PAH}$ patients to transition from bosentan to ambrisentan without influencing hematologic parameters or heart function and ambrisentan may be a viable treatment option for patients with PAH who had to discontinue bosentan due to different reasons.

Human subjects/informed consent statement: The study's protocol and ethics were approved by the ethics committee of the Second Xiangya Hospital of Central South University (No. ChiCTR1900025197) and was conducted in accordance with the Declaration of Helsinki. All patients who participated in this program agreed with the protocol and signed informed consent before study entry.

\section{Data Sharing Statement}

Data related to this manuscript are always available from the corresponding author upon reasonable request.

\section{Funding}

This work was supported by The National Natural Science Foundation of China (No. 81870233, 81600249), the Hunan Provincial Natural Science Foundation of China (No. 2017JJ3455). 


\section{Disclosure}

None of the authors report any conflicts of interest relevant to this subject.

\section{References}

1. Hoeper MM, Humbert M, Souza R, et al. A global view of pulmonary hypertension. Lancet Respir Med. 2016;4:306-322. doi:10.1016/ S2213-2600(15)00543-3

2. McGoon MD, Benza RL, Escribano-Subias P, et al. Pulmonary arterial hypertension: epidemiology and registries. J Am Coll Cardiol. 2013;62:D51-9. doi:10.1016/j.jacc.2013.10.023

3. Lau EMT, Giannoulatou E, Celermajer DS, Humbert M. Epidemiology and treatment of pulmonary arterial hypertension. Nat Rev Cardiol. 2017;14:603-614. doi:10.1038/nrcardio.2017.84

4. Sofer A, Ryan MJ, Tedford RJ, Wirth JA, Fares WH. A systematic review of transition studies of pulmonary arterial hypertension specific medications. Pulm Circ. 2017;7:326-338. doi:10.1177/2045 893217706357

5. Gu S, Hu H, Dong H. Systematic review of the economic burden of pulmonary arterial hypertension. PharmacoEconomics. 2016;34: 533-550. doi:10.1007/s40273-015-0361-0

6. Zhai Z, Zhou X, Zhang S, et al. The impact and financial burden of pulmonary arterial hypertension on patients and caregivers: results from a national survey. Medicine. 2017;96:e6783. doi:10.1097/ MD.0000000000006783

7. Zhang R, Dai LZ, Xie WP, et al. Survival of Chinese patients with pulmonary arterial hypertension in the modern treatment era. Chest 2011;140:301-309. doi:10.1378/chest.10-2327

8. Aypar E, Alehan D, Karagöz T, Aykan H, Ertugrul İ. Clinical efficacy and safety of switch from bosentan to macitentan in children and young adults with pulmonary arterial hypertension: extended study results. Cardiol Young. 2020;30:681-685. doi:10.1017/S10479511 20000773

9. Cadenas-Menéndez S, Álvarez VP, Oterino MA, et al. Evolution of patients with pulmonary arterial hypertension starting macitentan after the discontinuation of other endothelin-receptor antagonists: results of a Retrospective Study. Am J Cardiovasc Drugs. 2019.

10. Safdar Z, Thakur A, Frost A. Tolerability of switch to macitentan from bosentan in pulmonary arterial hypertension. South Med J. 2017;110:223-228. doi:10.14423/SMJ.0000000000000607

11. Ruiz MA, Pardo A, Rejas J, Soto J, Villasante F, Aranguren JL. Development and validation of the "Treatment satisfaction with medicines questionnaire" (SATMED-Q). Value in health: the. $j$ Int Soc Pharmacoecon Outcomes Res. 2008;11:913-926.
12. Galiè N, Humbert M, Vachiery J-L, et al. 2015 ESC/ERS Guidelines for the diagnosis and treatment of pulmonary hypertension. Eur Respir J. 2015;46:903. doi:10.1183/13993003.01032-2015

13. Condon DF, Nickel NP, Anderson R, Mirza S, de Jesus Perez VA. The 6th world symposium on pulmonary hypertension: what's old is new. F1000Research. 2019;8:8. doi:10.12688/f1000research.18811.1

14. Frantz RP, Durst L, Burger CD, et al. Conversion from sildenafil to tadalafil: results from the sildenafil to tadalafil in pulmonary arterial hypertension (SITAR) study. $J$ Cardiovasc Pharmacol Ther. 2014;19:550-557. doi:10.1177/1074248414528066

15. Lichtblau M, Harzheim D, Ehlken N, et al. Safety and long-term efficacy of transition from sildenafil to tadalafil due to side effects in patients with pulmonary arterial hypertension. Lung. 2015;193: 105-112. doi:10.1007/s00408-014-9657-7

16. Shapiro S, Traiger G, Hill W, Zhang L, Doran AK. Safety, tolerability, and efficacy of overnight switching from sildenafil to tadalafil in patients with pulmonary arterial hypertension. Cardiovasc Ther. 2013;31:274-279. doi:10.1111/1755-5922.12038

17. Tay EL, Geok-Mui MK, Poh-Hoon MC, Yip J. Sustained benefit of tadalafil in patients with pulmonary arterial hypertension with prior response to sildenafil: a case series of 12 patients. Int J Cardiol. 2008;125:416-417. doi:10.1016/j.ijcard.2007.01.046

18. Shlobin OA, Brown AW, Weir N, Ahmad S, Lemma M, Nathan SD. Transition of $\mathrm{PH}$ patients from sildenafil to tadalafil: feasibility and practical considerations. Lung. 2012;190:573-578. doi:10.1007/ s00408-012-9408-6

19. Benza RL, Mehta S, Keogh A, Lawrence EC, Oudiz RJ, Barst RJ. Sitaxsentan treatment for patients with pulmonary arterial hypertension discontinuing bosentan. J Heart Lung Transpl. 2007;26:63-69. doi:10.1016/j.healun.2006.10.019

20. McGoon MD, Frost AE, Oudiz RJ, et al. Ambrisentan therapy in patients with pulmonary arterial hypertension who discontinued bosentan or sitaxsentan due to liver function test abnormalities. Chest. 2009;135:122-129. doi:10.1378/chest.08-1028

21. Safdar Z. Effect of transition from sitaxsentan to ambrisentan in pulmonary arterial hypertension. Vasc Health Risk Manag. 2011;7:119-124. doi:10.2147/VHRM.S15026

22. Fox B, Langleben D, Hirsch AM, et al. Hemodynamic stability after transitioning between endothelin receptor antagonists in patients with pulmonary arterial hypertension. Can J Cardiol. 2013;29:672-677.

23. Gong SG, Wang L, Pudasaini B, et al. Transition from ambrisentan to bosentan in pulmonary arterial hypertension: a single-center prospective study. Canadian Respir J. 2018;2018:9836820. doi:10.1155/ 2018/9836820
International Journal of General Medicine

\section{Publish your work in this journal}

The International Journal of General Medicine is an international, peer-reviewed open-access journal that focuses on general and internal medicine, pathogenesis, epidemiology, diagnosis, monitoring and treatment protocols. The journal is characterized by the rapid reporting of reviews, original research and clinical studies across all disease areas. The manuscript management system is completely online and includes a very quick and fair peer-review system, which is all easy to use. Visit http://www.dovepress.com/ testimonials.php to read real quotes from published authors. 